\title{
Formale Rekonstruktion und vergleichende Rahmung soziologischer Theorien $^{1}$
}

\section{Jürgen Klüver}

Hochschuldidaktisches Zentrum, Universität Essen, Postfach 1037 64, D-4300 Essen

Zusammenfassung: In dem Beitrag wird ein systematisches Verfahren zur formalen Rekonstruktion soziologischer Theorien und zur Konstruktion eines Vergleichsrahmens für verschiedene Theorien vorgeschlagen. Ausgangspunkt ist die Mikro-Makro-Diskussion der theoretischen Soziologie: Soziologische Theorien werden als vertikal geschichtete Systeme angesehen, die sich aus mehreren Mikro- und Makroebenen (,Emergenzebenen") zusammensetzen. Das Verfahren wird illustriert an der Theorie des Historischen Materialismus. Gedacht ist dabei an den Einsatz spezieller wissensbasierter Programmsysteme.

„Die Gesellschaft besteht nicht aus Individuen, sondern drückt die Summe der Beziehungen aus, worin diese Individuen zueinander bestehen. “ (Marx)

„Die Vorstellung der Elemente des Gesellschaftssystems muß von substantiellen Einheiten (Individuen) auf selbstreferentielle Operationen umgestellt werden ... Dann ist unter Gesellschaft ganz einfach das umfassende soziale System aller aufeinander Bezug nehmenden Kommunikationen zu verstehen. (Luhmann) ${ }^{2}$

\section{Theorienvergleich und formale Rekonstruktionen}

Das Programm eines systematischen Theorienvergleichs hatte für die Soziologie in den siebziger Jahren eine gewisse Konjunktur, die ihren Auslöser vor allem in den mittlerweile schon fast klassischen Grundlagenkontroversen hatten (Positivismusstreit, Habermas vs. Luhmann). Nach einigen Zwischenergebnissen (vgl. insbesondere Hondrich/ Matthes 1978) stagnierten die Bemühungen jedoch

${ }^{1}$ Bei diesem Aufsatz handelt es sich um einen Teilbericht aus einem Forschungsprojekt, das seit ca. einem Jahr an der Universität - GH - Essen durchgeführt wird; Beteiligte sind außer mir Prof. Dr. Dieter Krallmann und Dr. Erhard Cremers vom Funktionsbereich Kommunikationswissenschaften. Es geht in dem Projekt darum, auf der Grundlage einer logischen Rekonstruktion soziologischer Theorien spezielle Programmsysteme, sog. wissensbasierte Systeme, zu entwickeln, so daß eine detaillierte Rekonstruktion und ein darauf basierender Vergleich soziologischer Theorien mit „Computerunterstützung“ durchgeführt werden kann (ähnlich spricht Hannemann (1988) von ,computer assisted theory building“). Die Gesamtkonzeption des Projekts ist dargestelit bei Klüver/Krallmann 1989; in diesem Aufsatz geht es vornehmlich darum, die ,,soziologische" Seite des Projekts einer kritischen Fachöffentlichkeit vorzustellen. Auf die Entwicklung und Operationsweise der wissensbasierten Systeme werden wir in weiteren Veröffentlichungen eingehen.

${ }^{2}$ Marx, Grundrisse der Kritik der politischen Ökonomie, Berlin 1953, S. 176; N. Luhmann, Ökologische Kommunikation, Opladen 1976, S. 24. ziemlich bald - aus welchen Gründen auch immer; statt dessen mehrten sich die kritischen Stimmen, die einen systematischen Theorienvergleich sowohl für undurchführbar als auch für überflüssig hielten.

Geradezu paradigmatisch wird die Skepsis gegenüber einem systematischen Theorienvergleich beispielsweise von Klinkmann (1981) formuliert: Im Fall des Vergleichs zweier Theorien, so die grundsätzliche Argumentation, gibt es strenggenommen nur zwei Möglichkeiten: Entweder sind die beiden Theorien tatsächlich verschieden - dann liegt das daran, daß sie sich an verschiedenen theoriestiftenden Paradigmen orientieren. Da diese gemäß Kuhn bekanntlich inkommensurabel sind, sind auch die beiden Theorien nicht vergleichbar, da es keinen Vergleichsrahmen geben kann. Die Theorien sind lediglich in ihrer paradigmatischen Verschiedenheit darzustellen und ansonsten beide in ihren jeweiligen Paradigmen $\mathrm{zu}$ belassen. Oder aber die Theorien sind lediglich Ausprägungen eines und desselben Paradigmas - dann sind sie strenggenommen gar nicht verschieden, sondern es wird sich erweisen, daß die eine Teil der anderen ist bzw. die eine Ergänzung der anderen etc. In beiden Fällen jedenfalls liegt kein Theorienvergleich im strengen Sinne vor; einen solchen kann es auch gar nicht geben.

Abgesehen davon, daß auch ein solches Ergebnis, nämlich ob es sich um Fall 1 oder 2 handelt, häufig alles andere als trivial ist, sind derartige a prioriArgumente im allgemeinen nicht sehr nahe an der Praxis des Wissenschaftsbetriebs. Zweifellos wer- 
den konkurrierende Paradigmen nicht durch logische Argumentationen etabliert oder verworfen das wußte bekanntlich schon Max Planck. Dennoch wurden und werden ständig Theorien miteinander verglichen; die wissenschaftssoziologisch häufig konstatierte Tatsache, daß gewöhnlich nicht unbedingt logisch zwischen Theorien und schon gar nicht zwischen Paradigmen entschieden wird, besagt ja nicht, daß man eine Unterscheidung und zuweilen auch Entscheidung zwischen konkurrierenden Theorien nicht treffen kann. ${ }^{3}$ Kuhn selbst unterlag hier, wie mir scheint, einer klassischen „naturalistic fallacy“.

Wie dem auch sei, hier soll über diese Probleme nicht weiter verhandelt werden. Unbeschadet der unleugbaren Probleme eines systematischen Theorienvergleichs, der in den Sozialwissenschaften auch sicher wesentlich mühsamer ist als in den von Kuhn analysierten Naturwissenschaften, lehrt die Lektüre jedes soziologischen Theoriebuchs, daß nun einmal ständig Theorienvergleiche durchgeführt werden und natürlich für jeden anspruchsvollen theoretischen Diskurs auch völlig unverzichtbar sind. Die Frage ist deswegen auch nicht, ob Theorienvergleiche möglich und notwendig sind dies beantwortet der institutionalisierte Wissenschaftsbetrieb täglich affirmativ. ${ }^{4}$ Die Frage ist vielmehr, wie ein solches Unternehmen durchgeführt werden kann, so daß die Unterschiedlichkeit der Theorien nicht von vornherein durch die Wahl des Vergleichsrahmens entweder verwischt wird oder die Überlegenheit einer Theorie über die andere automatisch feststeht.

Die beiden zu Beginn aufgeführten Zitate verdeutlichen vermutlich bereits, in welcher Hinsicht hier ein Theorienvergleich konzipiert wird: Es geht um formale Konstitutions- und Konstruktionsprinzipien von allgemeinen soziologischen Theorien, die zum Gegenstand eines Vergleichs gemacht werden sollen - nicht um Intentionen, ideologische Implikationen, politische Kontexte u.ä. Bildlich gesprochen geht es also um den Vergleich von Theoriearchitekturen und erst einmal auch nur um diese. So gehört zur Architektur einer Theorie zweifellos die theoriespezifische Konstitution des Gegenstandsbereichs der Theorie - offenbar sind in dieser Dimension der Theoriearchitektonik die Theorien von Luhmann und Marx nicht nur vergleichbar, sondern zumindest äquivalent: sie favorisieren beide eine dezidierte Abstraktion vom Individuum.

Die Architektonik einer Theorie ergibt sich aus der Theorie im allgemeinen nicht unmittelbar. Soziologische Theorien stellen sich den Rezipienten als hochgradig unformale Gebilde dar, deren logische Struktur häufig selbst Gegenstand langwieriger Debatten ist. Will man also soziologische Theorien hinsichtlich ihrer formalen Struktur miteinander vergleichen, ist erst einmal diese Struktur herauszuarbeiten; diese Reduktion von Theorien auf einen logischen Strukturkern ist folglich der systematisch erste Schritt, der beim Vergleich von Theorien durchzuführen ist und der im folgenden als Rekonstruktion bezeichnet werden soll. ${ }^{5}$ Dabei geht es natürlich um eine sehr gezielte Reduktion: Leitend ist hier die Annahme, daß es für soziologische Theorien überhaupt eine strukturelle Gemeinsamkeit gibt, die einerseits allgemeiner ist als die Struktur jeder einzelnen Theorie, andererseits für alle Theorien die strukturelle Repräsentation ihrer gemeinsamen Funktion ist, soziale Realität kognitiv zu erschließen. Wenn sich eine solche Annahme als plausibel herausstellt, dann wären die Strukturkerne der einzelnen Theorien - ihre Architekturen - gewissermaßen als Variationen zum gemeinsamen Strukturthema aufzufassen. Einen Vorschlag für ein derartiges Strukturthema werde ich im übernächsten Abschnitt skizzieren. ${ }^{6}$

\footnotetext{
${ }^{5}$ Ich lehne mich bei der Verwendung des Begriffs „Rekonstruktion" bewußt an den Sprachgebrauch bei Habermas (1976) an, da es mir auch um ,auseinandernehmen" und „wieder zusammensetzen“" geht, wenn auch freilich mit einer sehr anderen Intention als die von Habermas.

${ }^{6}$ Wenn z. B. Hondrich (1978: 324f) in der Soziologie „viele theoretische Ansätze, aber nur eine soziologische Theorie" sieht, scheint er m.E. einen ähnlichen Gedanken ausdrücken zu wollen: „Die eine soziologische Theorie" basiert auf einer strukturellen Gemeinsamkeit der ,vielen theoretischen Ansätze“.
} 


\section{Rekonstruktions- und Interpretationsprobleme}

Wenn man versuchen wollte, formal exakt zu bestimmen, was eine soziologische Theorie ist, müßte man wahrscheinlich die Ergebnisse formaler Rekonstruktionsprozesse vorwegnehmen; einschlägige Versuche in wissenschaftstheoretischen Handbüchern zeigen auch, wie stark derartige begriffliche Festlegungen von den jeweiligen wissenschaftstheoretischen Grundpositionen der Autoren abhängig sind (exemplarisch Cohen 1980; Bayer/Stölting 1989). Einigkeit besteht noch nicht einmal darüber, was alles unter diesen Begriff fallen soll: Ein an der analytischen Wissenschaftstheorie orientierter Autor wie Cohen läßt gemäß seinen Standards eigentlich nur die Theorie von Parsons gelten; bei nicht so restriktiver Sicht (Bayer/Stölting) bleibt nichts anderes übrig, als einfach von Marx bis zum Behaviorismus alle wichtigen Traditionen zu nennen.

Bei einer derartigen Unklarheit über den Gegenstand ,soziologische Theorie“ geht es wohl nur, verhältnismäßig pragmatisch vorzugehen: Es gibt offensichtlich kognitive Gebilde, die von professionellen Soziologen immer dann genannt und rezipiert werden, wenn es in der Soziologie um „Theorie“ geht. Gemeint sind damit gewöhnlich die sogenannten Klassiker und ihre jeweiligen Schulen, also von Marx angefangen bis hin zu Luhmann und Habermas. In der soziologischen scientific community existiert also so etwas wie ein Minimalkonsens hinsichtlich eines extensionalen Kerns des Begriffs „soziologische Theorie“, der ansonsten gemäß dem berühmten Wittgensteinschen Prinzip nur über „Familienähnlichkeiten“ zusammengehalten wird.

Bei unseren Rekonstruktions- und Vergleichsversuchen werde ich mich gemäß dieser pragmatischen Festlegung verhalten: Gegenstand dieser Analysen sind Theorien, die zum „extensionalen Kern" gehören. Aus forschungspraktischen Gründen konzentrieren wir uns gegenwärtig auf die Theorien der eingangs zitierten Autoren, also Marx und Luhmann; in diesem Aufsatz werde ich unser Vorgehen vor allem an der Theorie von Marx illustrieren. Dies hat seinen Grund auch darin, daß die Theorie von Marx wie keine andere Theorie verhältnismäßig häufig Gegenstand sehr unterschiedlicher Rekonstruktionsversuche gewesen ist und sich aufgrund ihrer Struktur auch dafür anbietet (für z.T. sehr formale Rekonstruktionen, die unseren Intentionen verwandt sind, vgl. vor allem Diederich 1981 und Hannemann 1988 sowie
Hannemann/Collins 1986). Der (analytische) Paradigmacharakter der Marxschen Theorie für soziologische Theoriebildung überhaupt scheint mir unbeschadet einer ideologiekritischen Bewertung der Rezeptionsgeschichte dieser Theorie hinreichend Grund dafür zu sein, Analysen zur Theoriebildung in der Soziologie immer wieder auch an dem Klassiker Marx zu orientieren.

Versucht man nun, derartige Theorien unter formalen Gesichtspunkten näher zu analysieren, dann fällt sofort auf, da $\$$ es sich dabei um keineswegs einheitliche Gebilde handelt. Damit ist noch gar nicht der (inhaltliche) Aspekt gemeint, daß gerade bei Theoretikern mit einer lebenslangen Forschungs- und Publikationspraxis häufig bestritten wird, daß es sich beim Gesamtwerk überhaupt um ein einheitliches Theoriengebäude handelt (,alter versus junger Marx"). Gemeint ist der formale Sachverhalt, daß diese Theorien, soweit sie als sprachlich formulierte Komplexe betrachtet werden, aus Aussagen bzw. Sätzen ${ }^{7}$ sehr unterschiedlicher Provenienz zusammengefügt sind. In einer sehr groben Klassifikation lassen sich mindestens folgende Aussagentypen unterscheiden:

(a) Sätze, die die Theorie strategisch plazieren, also den theoretischen Diskurs aus der Sicht des Autors thematisieren - polemisch, zustimmend, sich absetzend etc. Vor allem die Marxschen Frühwerke sind, wie bekannt, voll von derartigen Sätzen, was ebenso für die Luhmannschen Texte gilt (man lese daraufhin die Einleitung zu Luhmann 1984). Es gehört schlicht zum Geschäft, sich als Theoretiker selbst im theoretischen Kosmos der Soziologie zu plazieren, was z. B. in den Naturwissenschaften eher implizit geschieht. In der Dimension einer logischen Semantik handelt es sich hier offenbar um metatheoretische Sätze.

(b) Ebenfalls als metatheoretische Sätze sind solche Aussagen zu bestimmen, die auf außerwissenschaftliche Bezüge der Theorie aufmerksam machen sollen - Verweise auf „Praxis“, „Aufklärung“ oder „Ideologiekritik“, „gesellschaftliche Steuerung" etc. Natürlich kann man, wie vor allem die Vertreter der Kritischen Theorie, darauf insistieren, daß bestimmte Theorietypen gerade durch derartige außerwissenschaftliche Beziehungen charakterisiert sind - das „Kritische“ an der Kritischen Theorie. Unter analytischen Aspekten jedoch sind

\footnotetext{
${ }^{7}$ Ich sehe hier bewußt von dem logisch bzw, sprachphilosophisch wohl begründeten Unterschied zwischen „Sätzen“ und „Aussagen“ ab, da dieser hier keine Rolle spielt.
} 
dies zusätzliche Dimensionen zum Kernbereich der Theorie, nämlich der konstituierenden und erklärenden Funktion der Theorie.

(c) Nur zu erwähnen ist hier schließlich eine dritte Kategorie von metatheoretischen Sätzen, nämlich „didaktischen“ Aussagen, die die Rezeption der Theorie erleichtern sollen.

(d) Die „eigentlich" zur Theorie gehörenden Sätze, aus deren Gesamtheit sich die Architektur der Theorie ergibt, bilden auch noch keine homogene Klasse; es lassen sich mindestens zwei Typen unterscheiden, von denen der erste Typus in den beiden Zitaten zu Beginn repräsentiert wird. Dabei handelt es sich um Aussagen, die sich in der Theorie auf deren erkenntnistheoretische und logische Struktur beziehen; u.E. sind dies Bestandteile der Theorie selbst, auch wenn sie sich nicht unmittelbar auf den Gegenstandsbereich der Theorie beziehen, sondern strenggenommen ebenso wie die Typen (a)-(c) die Theorie thematisieren. Jede komplexe soziologische Theorie jedoch weist diesen Doppelcharakter auf, nämlich sich mit den gleichen logischen Grundkategorien gleichzeitig fremdreferentiell auf Gegenstandsbereiche und selbstreferentiell auf sich selbst zu beziehen. ${ }^{8}$

(e) Schließlich ist hier die quantitativ natürlich dominierende Menge der Sätze zu nennen, die sich als theoretische Konstituierungen und vor allem Erklärungen eines sozialen Bereichs verstehen lassen. Das sind Aussagen sehr unterschiedlicher Reichweite, die dazu manchmal in Typus (d) „changieren“ - z.B. das berühmte Marxsche Diktum, daß die Geschichte eine Geschichte der Klassenkämpfe ist. Eine Feinanalyse dieses letztlich entscheidenden Typus wird $\mathrm{zu}$ den wichtigsten Aufgaben von formalen Rekonstruktionen gehören.

Rezipiert werden soziologische Theorien im allgemeinen als Gesamtgebilde, bei denen nicht unbedingt zwischen den verschiedenen Aussagentypen unterschieden wird. Entsprechend heterogen laufen dann auch häufig die Diskurse über die Theorien $\mathrm{ab}$. Eine formale Rekonstruktion hat damit

\footnotetext{
${ }^{8}$ Ein Beispiel dafür ist die duale Verwendung der Begriffe "Lebenswelt" und "System" bei Habermas (1981 II), was Habermas sehr pointiert hervorhebt (Habermas 1986). Angelegt ist dies bekanntlich schon bei Kant: „Die Bedingungen der Möglichkeit der Erfahrung überhaupt sind zugleich Bedingungen der Möglichkeit der Gegenstände der Erfahrung." (Kritik der reinen Vernunft, A. 158)
}

die Aufgabe, von sehr vielen Konnotationen zu abstrahieren, mit denen die Theorien durch ihre Formulierung und ihre entsprechende Rezeption versehen sind; die Rekonstruktion muß sich ausschließlich auf die Aussagentypen (d) und (e) konzentrieren.

Wenn man voraussetzt, daß eine derartige Unterteilung in verschiedene Aussagentypen im Einzelfall auch durchzuführen ist, dann ergibt sich freilich sofort das Problem, ob damit nicht Aspekte der Theorie verloren gehen, die für viele Rezipienten das eigentlich Wertvolle an der Theorie ausmachen. Anders ausgedrückt: Unterscheiden sich vielleicht sozialwissenschaftliche Theorien von naturwissenschaftlichen Theorien gerade (positiv) dadurch, daß auf ihre „metatheoretischen“ Aspekte gar nicht verzichtet werden kann, wenn das Ergebnis nicht trivial sein soll?

Die Diskussionen über dieses bekannte Problem sind in unterschiedlichsten Formulierungen bekanntlich uferlos und vielleicht auch gar nicht wissenschaftsimmanent $\mathrm{zu}$ lösen. Im Positivismusstreit (Adorno 1970) ging es anscheinend vor allem um dieses Problem und vielleicht war dies auch der Grund dafür, daß die Versuche um systematische Theorienvergleiche letztlich unfruchtbar blieben. Die in Lepsius 1976 dokumentierten Grundlagenkontroversen lassen sich auch als Variationen zu diesem offensichtlich unerschöpflichen Thema lesen.

Ich will dieses Problem weder entscheiden noch leugnen. Damit jedoch überhaupt ein Fortschritt in der Hinsicht möglich ist, Einsichten in strukturelle Gemeinsamkeiten verschiedener Theorien zu gewinnen, und auf diesem Weg möglicherweise Theorieentwicklungen selbst in formaler Hinsicht zu befruchten, scheint es mir unumgänglich, hier eine bewußte Reduktion der so nicht handhabbaren Gesamtkomplexität vorzunehmen. Unabhängig von dem genannten Problem ist wohl unstrittig, $d a \beta$ es analytische Kerne jeder anspruchsvollen soziologischen Theorie gibt und daß diese als solche formal analysiert werden können. Beschränkt man sich auf dieses Problem - das immer noch komplex genug ist -, dann geht vielleicht einiges von dem verloren, was in der Wirkungsgeschichte das eigentlich Faszinierende an den Theorien ausgemacht hatte und ausmacht. In einer formal nicht handhabbaren Ganzheit sind derartige überkomplexe Gebilde allerdings wohl nur noch als kulturelle Phänomene zu verstehen, was eine sicher nicht reizlose Betrachtungsweise ist. Geht es jedoch darum, theoretischen Fortschritt einer Diszi- 
plin auch als Gewinn an begrifflicher Präzision und struktureller Transparenz zu realisieren, bleibt wohl nur der Weg einer bewußten und begründbaren Reduktion.

\section{Metatheoretische Rekonstruktionen: Makro und Mikro}

Metaphorisch gesprochen lassen sich theoriespezifische Strukturen als Rahmungen (frameworks vgl. Carnap 1950; Minsky 1975) für den Inhalt der Theorien auffassen, wobei im Gegensatz zu Bilderrahmen allerdings durch die strukturellen Rahmungen kategoriale Konstitutionen der Inhalte erfolgen. Entsprechend kann man sich die Suche nach strukturellen Gemeinsamkeiten unterschiedlicher Theorien als Konstruktion einer metatheoretischen Rahmung vorstellen, für die die theoriespezifischen Strukturen dann "Inhalte“ sind. Damit durch eine solche metatheoretische Rahmung jedoch keine zu engen Festlegungen erfolgen, die jeden Theorievergleich dann unzulässig präjudizieren müßten, kann es sich nur um eine sehr allgemeine Rahmung handeln, die sich aus prinzipiellen Annahmen über Funktion und Struktur soziologischer Theoriebildung begründen lassen muß. M.E. ergeben sich derartige Annahmen aus einer theoretischen Diskussion, die unter dem Signum „Mikro- versus Makrotheorien“ in der Soziologie geführt wird (vgl. insbesondere Knorr-Cetina/Cicourel 1981; Alexander et al. 1987; Fielding 1989). Ich verstehe die hier entwickelte metatheoretische Rahmung, also den Vergleichsrahmen der einzelnen Theorien, als Verallgemeinerung der (vorläufigen) Ergebnisse dieser und anderer Diskussionen. Diese Ergebnisse müssen freilich selbst etwas präziser rekonstruiert werden.

Begriffe wie Mikro(soziologie) und Makro(soziologie) suggerieren auf einen ersten Blick, daß es sich dabei jeweils um homogene Positionen handelt, die sich entsprechend einfach charakterisieren lassen. Auf einem allgemeinen Abstraktionsniveau ist dies auch nicht falsch: Wenn man eine sehr plastische Formulierung von Luhmann verwendet (Luhmann 1984: 43), dann kann man sagen, daß Makrotheorien ihren Gegenstandsbereich ,von oben konstituieren“, Mikrotheorien dagegen „von unten". Bei den daran anschließenden Fragen freilich, was denn nun jeweils „oben“ und „unten“ ist und was es für die Realität sozialer Phänomene bedeutet, wenn Theorien von oben oder unten "konstituieren“, scheiden sich sehr rasch die Positionen und durchaus heterogene Konzepte werden sichtbar (vgl. die einschlägigen Übersichten bei Alexander et al. 1987, Giesen 1987 und Münch/ Smelser 1987).

Hierzu nur einige Beispiele, um diese Heterogenitäten zu verdeutlichen: Die klassische Formulierung der Mikro-Makro-Distinktion ist häufig die Unterscheidung zwischen Handlung und Struktur (,Actions and Structure" nennt Fielding demnach seinen Band zur Mikro-Makro-Debatte). Von ,unten" gesehen wäre also Handlung der Begriff, der die analytische Ausgangsebene kennzeichnet und wohl auch die empirische Basis. Viele Mikrotheoretiker würden dem wohl zustimmen; entsprechend würden viele Makrotheoretiker wohl konzedieren, daß Handlung die für sie unterste Ebene als empirische Bestätigungsinstanz charakterisiert. Das ist jedoch keinesfalls für alle Mikro- oder Makrotheoretiker zwingend. Opp und Wippler beispielsweise sprechen in diesem Zusammenhang von "Individualtheorien, die das Verhalten von individuellen Akteuren im sozialen Kontext erklären" können (Opp/Wippler 1990: 9). Die Mikroebene als analytischer Bezugspunkt scheint hier eher das einzelne Individuum zu sein; entsprechend gehen auch makrotheoretisch orientierte Systemtheoretiker wie Krohn und Küppers (1989) bei ihrer Analyse des Wissenschaftssystems davon aus, daß Individuen Elemente sozialer Systeme sind. ${ }^{9}$ Noch anders bestimmen Ansätze in der Tradition des symbolischen Interaktionismus die Interaktion, also nicht die isolierte Handlung als analytische und empirische Basis ihrer theoretischen Modelle; „Mikro“ ist also hier stets in terms von (relationaler) Reziprozität konzipiert. An die Figur der Habermasschen und Apelschen Transzendentalpragmatik sei in diesem Zusammenhang nur erinnert.

Entsprechend heterogen ist die Bestimmung von „Makro“. Mikrotheoretiker, die häufig sogar die Möglichkeit bestreiten, auch nur sinnvoll von eigenständigen Makroebenen zu sprechen, konzedieren in diesem Zusammenhang gewöhnlich bestenfalls Aggregationen von Handlungen oder auch Individuen; situationsübergreifende Strukturen sind dann höchstens als Vernetzung unintendierter Handlungsfolgen zu verstehen (vgl. KnorrCetina 1981 sowie die Beiträge in Knorr-Cetina/ Cicourel 1981). Doch auch bei Theoretikern, die

\footnotetext{
${ }^{9} \mathrm{Vgl}$, auch die ,individualtheoretische“ Position bei Esser 1990, der darin eine der wesentlichen Gemeinsamkeiten zwischen Schütz und dem rational choice Ansatz sieht.
} 
explizit über reine Mikroansätze hinausgehen, ist die als logische Bezugsebene fungierende Makroebene gewöhnlich nicht einheitlich zu anderen Ansätzen festgelegt: Zuweilen ist dies die „Gesellschaft" - so jedenfalls bei klassischen Gesellschaftstheorien -, zuweilen Institutionen oder auch Organisationen, manchmal auch größere Gruppen von Individuen etc. Was in diesem Zusammenhang logisch möglich ist, findet sich auch in der allgemeinen Diskussion.

Die hier skizzierten begrifflichen und theoretischen Heterogenitäten machen es offenbar unmöglich, sich anders als durch dezisionistische Optionen unmittelbar einem der verschiedenen Ansätze zuzuordnen. Deshalb liegt es nahe, eine Generalisierung der herkömmlichen Mikro-Makro-Distinktion vorzunehmen, um daraus allgemeine Struktureigenschaften soziologischer Theoriebildung $\mathrm{zu}$ erhalten. Dies soll im folgenden aufgrund zweier Prämissen durchgeführt werden:

Die erste Annahme postuliert, daß die MikroMakro-Distinktion nur dann fruchtbar gemacht werden kann, wenn sie nicht als Dichotomie begriffen wird, bei der Orientierungen an einer Seite die andere ausschließen, sondern wenn sie als Charakterisierung zweier Pole verstanden wird, die nur gemeinsam und in ihren Beziehungen zueinander sinnvoll sind. Das scheint gegenwärtig ein weithin akzeptierter Konsens zu sein (vgl. Münch/ Smelser 1987). Im Zusammenhang mit dieser ersten Annahme ergibt sich, daß die Mikro-MakroDistinktion vor allem unter ihrem analytischen Aspekt, also als vorwiegend analytische Distinktion, verstanden werden sollte (vgl. dazu insbesondere Alexander 1987). Dies wird im folgenden noch deutlicher. Bei der zweiten Annahme gehe ich davon aus, daß die traditionelle Unterscheidung in jeweils eine Mikro- und Makroebene zu eng ist und erweitert werden muß durch eine Unterscheidung verschiedener Ebenen - im allgemeinen mehr als zwei -, die paarweise zueinander in einer Beziehung stehen, die der traditionellen Mikro- und Makrodifferenzierung entspricht. Diese Annahme erlaubt es, die verschiedenen Bestimmungen von jeweils „Mikro“ und „Makro“ nicht als Alternativen zu betrachten, sondern sie selbst als Teile eines allgemeineren Ganzen zu sehen.

Betrachtet man klassische Makrotheorien - z.B. die des Historischen Materialismus - und etablierte Mikroansätze - etwa in der Tradition des symbolischen Interaktionismus -, dann zeigt sich auf einer sehr formalen Ebene folgende gemeinsame „Theoriearchitektonik“ (Luhmann): Analytische
Basis (nicht notwendig die empirische) ist stets eine bestimmte Begriffsebene, mit der die Theorie ihren logischen Anfang nimmt. (Im anders gelagerten Fall der Mathematik sind dies die Axiome.)

Die analytische Basis einer soziologischen Theorie charakterisiert im ersten Fall eine sehr allgemeine Gesellschaftsstruktur: dann handelt es sich um eine Makrotheorie, deren spezifische Erklärungsleistung darin besteht, soziale Phänomene unterschiedlicher Allgemeinheit in den begrifflich-kategorialen Rahmen der Theorie einzuordnen und die Phänomene auf generell sehr komplexe Weise aus den Annahmen „abzuleiten“, die als Beziehungen zwischen den Begriffen auf der Basisebene, also sozusagen den Basisbegriffen, formuliert werden. Im zweiten Fall wird durch die analytische Basis eine Mikroebene festgelegt, die z.B. als Handlungs- oder Interaktionsebene bestimmt wird. Die Leistung der Theorie besteht dann darin, einmal die alltäglich erfahrbare Realität in terms der Basisebene zu rekonstruieren und damit auf allgemeinere Handlungs- oder Interaktionsprinzipien zurückzuführen; zum anderen muß zumindest programmatisch gezeigt werden, wie allgemeinere Sachverhalte - „Makrophänomene“ - als Produkte der Basisebene dargestellt und somit erklärbar gemacht werden können - als Aggregationen, Vernetzung unintendierter Handlungsfolgen u.ä. In diesem Fall ist die Basisebene gleichzeitig die empirische Basis; im ersten Fall gilt das ex definitione nicht. In beiden Fällen jedoch muß die Theorie zeigen, wie durch den Ausgang von der Basisebene die andere oder die anderen Ebene(n) begrifflich erfaßt und erklärt werden können. ${ }^{10}$

Am Beispiel wohl der klassischen Makrotheorie, der des Historischen Materialismus, kann dies etwas verdeutlicht werden. Der für die gesamte Theorie konstitutive Basisbegriff ist zweifellos der der Arbeit, der als ,produktive Wechselwirkung mit der Natur" die Gattung Mensch charakterisiert (vgl. z.B. Lange 1980). "Arbeit" ist demnach streng genommen ein protosoziologischer Begriff, da durch ihn „nur“ eine differentia specifica der biologischen Gattung bestimmt wird, die Analyse-

${ }^{10}$ Der Begriff „Erklären“ ist hier noch nicht unbedingt in einem strengen logischen Sinne, etwa in der Bedeutung des berühmten Hempel-Oppenheim Schemas, zu verstehen. Für viele - die meisten - soziologischen Theorien wäre dies eine zu enge Begriffsverwendung, da „Erklären“ dort im allgemeinen nur umgangssprachlich formulierte Beziehungen zwischen Aussagen unterschiedlicher Allgemeinheitsstufe bedeutet. Vgl. dazu beispielsweise Opp/Wippler 1990. 
ebene des Sozialen im allgemeinen Sinn mithin noch nicht erreicht ist. ${ }^{11}$ Aus der Arbeitskategorie ergibt sich dann erst die Basisebene der Theorie, nämlich die Begriffsdualität Produktivkräfte und Produktionsverhältnisse sowie die bekannte (dialektische) Spannungs- und Adäquatsheitsbeziehung zwischen ihnen. Damit ist strukturell festgelegt, wie soziale Phänomene von der Theorie erfaßt werden. Allerdings ist mit dieser Basisebene natürlich noch nichts über Gesellschaft in irgendeiner Konkretion gesagt; dazu bedarf es der Einführung weiterer Begriffe, die dann deutlich immer konkretere Sachverhalte beschreiben. Die Einteilung in Basis und Überbau ist eine schon konkretere Bestimmung von Gesellschaft, da hier bereits auf einzelne soziale Handlungsbereiche Bezug genommen wird - Ökonomie einerseits, Recht, Religion, Politik etc. andererseits. Die Einführung des Klassenbegriffs, die ebenso wie die Basis-Überbaubestimmung kategorial aus der Basisstruktur folgt - der ,materialistische" Charakter der Theorie -, ist eine weitere Konkretisierung, nämlich sowohl eine Festlegung politischer Grundstrukturen als auch eine Bestimmung der Prägung des individuellen Bewußtseins („Klassenbewußtsein“). Dies ließe sich fortsetzen. Wesentlich an diesem Rekonstruktionsbeispiel ist jedenfalls, daß sich einerseits die unterschiedlichen Begriffe in ihrer theorieimmanenten Funktion streng aus den Festlegungen der Basisebene ergeben, andererseits die verschiedenen Begriffe sehr deutlich unterschiedliche Ebenen der theoretischen Analyse erfassen. In reifizierenden Sprechweisen wäre dies die Erfassung unterschiedlicher Ebenen der Gesellschaft. Bereits hier muß betont werden, daß diese Schritte von der ,anthropologischen“ Kategorie der Arbeit bis zur begrifflichen Bestimmung der gesellschaftlichen Präformation individuellen Bewußtseins zwar streng der Architektonik der Theorie folgen - sie konkretisieren in wachsendem Maße den materialistischen Charakter der Theorie -, aber auf keinen Fall mit logischen Deduktionen verwechselt werden dürfen. Das Verhältnis zwischen den Begriffen und entsprechenden Theoriesätzen einer Ebene zu denen anderer Ebenen muß vielmehr so gedeutet werden, da 3 die jeweils konkretere Ebene aufzeigt, wie sich die abstrakteren Zusammenhänge auf den "vorhergegangenen“ Ebenen in konkrete-

${ }^{11}$ Zum Konzept der Protosoziologie vgl. Luckmann 1980. Das Konzept einer „Protowissenschaft“" ist allgemein eingeführt und entwickelt worden in den Arbeiten der sog. Erlanger Schuler, so z.B. bei Lorenzen 1962 und Janisch 1969. ren Zusammenhängen reproduzieren. Da es sich um konkretere Zusammenhänge handelt, ist die konkretere Ebene auch inhaltlich ,reicher" als die abstraktere und kann sich nicht als Deduktionsergebnis verstehen lassen. So folgt aus der Basisebene, daß die Einteilung der Menschen in einer bestimmten Gesellschaft sich als Konkretisierung der Basisebene „irgendwie“ aus dem Begriffspaar Produktivkräfte/Produktionsverhältnisse ergeben muß, und dafür ist der Klassenbegriff offensichtlich hervorragend geeignet. Wie nun jedoch die Klassenbeziehungen in einer bestimmten Gesellschaftsformation die politische Geschichte bestimmen und welche Ausformungen ein bestimmtes Klassenbewußtsein ausweist, ergibt sich selbstverständlich nicht aus der Basisebene: In Relation zur Basisebene sind derartige Sachverhalte „empirisch kontingent".

Verallgemeinert man dies Beispiel, dann gelangt man zur folgenden Grundstruktur von Theorien die Rede ist hier von idealtypischen Theorien, die in noch zu präzisierender Weise als strukturell vollständig bezeichnet werden können:

Soziologische Theorien lassen sich strukturell verstehen als vertikal geschichtete Systeme; die Schichtungen werden hier als Ebenen bezeichnet. Es gibt stets eine logisch primäre Ebene, die Basisebene, die kategorial den weiteren Aufbau der Theorie festlegt. Neben der Basisebene gibt es mindestens eine weitere Ebene - im allgemeinen enthält die Theorie mehr als eine weitere Ebene-, auf der die sozialen Phänomene thematisiert werden, die durch die Basisebene „erklärt“ werden (vgl. Anm. 10). Falls nur zwei Ebenen existieren, liegt eine Parallele vor zur klassischen Unterscheidung zwischen Explanans - die Basisebene - und dem Explanandum - die zusätzliche Ebene. Strukturell differieren Theorien in zwei Dimensionen: Sie haben unterschiedliche Reichweiten in vertikaler Hinsicht, d. h. sie enthalten mehr oder weniger viele Ebenen, und sie konstituieren „von oben“ oder von „unten“, d.h. sie gehen makrotheoretisch oder mikrotheoretisch vor. Wenn eine Theorie so viele Ebenen enthält, daß alle analytisch unterscheidbaren sozialen Gegenstandsbereiche durch die Theorie erfaßt werden können, dann ist die Theorie strukturell vollständig; im Sinne Luhmanns (1984: 9) handelt es sich dann um eine Theorie mit Universalitätsanspruch.

Zwischen den einzelnen Ebenen besteht generell kein deduktionslogisches Verhältnis: Die Basisebene legt kategorial zwar fest, welche Konstitutionsprinzipien für die anderen Ebenen zu gelten 
haben, aber sie kann dies nicht inhaltlich. Man kann die vertikale Ebenenschichtung der Theorie durch eine einfache formale Notation präzisieren: Bezeichnet man die Basisebene als $\mathrm{E}_{1}$, dann hat die Ebene, die unmittelbar von der Basisebene bestimmt wird, die Bezeichnung $\mathrm{E}_{2}$, die Ebene, die mittelbar über $E_{2}$ von der Basisebene bestimmt wird, ist $E_{3}$ und so fort. Im Fall des Historischen Materialismus wäre also die (protosoziologische) Basisebene die mit „Arbeit" charakterisierte $E_{1}$, die „nächste" Ebene die der Produktionsverhältnisse und Produktivkräfte $E_{2}$, „Basis und Überbau" charakterisieren $E_{3}$, der Klassenbegriff $E_{4}$ etc. Wie im Fall des Historischen Materialismus konstituiert $E_{1}$ alle Ebenen, aber mit Ausnahme von $E_{2}$ geschieht dies bei $E_{3}$ bis $E_{n}$ stets vermittelt über die dazwischen liegenden Ebenen; diese fügen der Theorie jeweils sowohl neue Konkretionen als auch neue ebenenspezifische Konstitutionsprinzipien hinzu, die natürlich kompatibel mit denen von $E_{1}$ sein müssen. In diesem Sinne habe ich beim Beispiel des Historischen Materialismus davon gesprochen, daß es im Verhältnis der Ebenen zueinander den Aspekt der ,empirischen Kontingenz“ gibt: $E_{k+1}$ enthält in Relation zu $E_{k}$ (und allen Ebenen $E_{i}$ mit $i<k$ ) empirisch kontingente Teile in dem Sinn, daß die sozialen Phänomene, die auf $\mathrm{E}_{\mathrm{k}+1}$ thematisiert werden, sowohl durch die Konstitutionsprinzipien und Erklärungen der Ebenen $\mathrm{E}_{1}$ bis $\mathrm{E}_{\mathrm{k}}$ als auch durch Konstitutions- und Erklärungsprinzipien, die spezifisch sind für $E_{k+1}$, erfaßt werden müssen. ${ }^{12}$ Etwas weniger formal läßt sich dies auch dadurch ausdrücken, daß auf jeder neuen Ebene neue logische Prinzipien der Theorie entwickelt werden müssen - sie „tauchen auf“" um eine Metapher zu benutzen. Von daher bietet es sich an, in einer mittlerweile seit spätestens Parsons bekannten Terminologie von Emergenzebenen der Theorie zu sprechen; paarweise verhalten sich zwei Emergenzebenen $E_{k}$ und $E_{k+1}$ zueinander als Mikro- und Makroebene: $E_{k}$ ist Mikroebene für $E_{k+1}$ (und $E_{k+1}$ Makroebene $z u E_{k}$ ), wenn es sich bei der Theorie um eine Mikrotheorie handelt; im Makrofall ist natürlich $\mathrm{E}_{\mathrm{k}}$ Makroebene für $\mathrm{E}_{\mathrm{k}+1}$ und umgekehrt. ${ }^{13}$

Einige zusätzliche Hinweise sind hier erforderlich: Die hier skizzenhaft dargestellte strukturelle Theorienrekonstruktion macht selbstverständlich keine

12 Strenggenommen ist der Begriff der empirischen Kontingenz nur bei Makrotheorien sinnvoll, da hier bei einem Ebenenübergang $E_{k}$ zu $E_{k+1}$ ein Gewinn an Empirie vorliegt. Das ist offensichtlich bei Mikrotheorien so nicht der Fall (s. u.).
Annahmen über die „,reale Existenz“ der einzelnen Ebenen. Die Behauptung ist hier demnach nicht die, daß „die Gesellschaft" aus verschiedenen Ebenen besteht, die als solche alle mehr oder weniger real wären. Dies ist z.B. die Position des sog. Mehrebenenmodells der Sozialisationstheorie (vgl. Geulen/Hurrelmann 1980). Hier wird „lediglich“ postuliert, daß jede soziologische Theorie, die diesen Namen verdient, eine derartige vertikale Schichtung als ihre "Theorienarchitektur" aufweist, wobei es wohl von Theorie zu Theorie verschieden ist, welcher Realitätsgehalt den einzelnen Ebenen zuerkannt wird. Es gibt z.B. sicher viele dem Historischen Materialismus verpflichtete Theoretiker, die alle hier genannten Ebenen als real ansehen würden. Die logisch allgemeinste Sichtweise ist für unsere Belange jedoch die, daß jede Theorie derartige analytische Distinktionen machen muß und faktisch auch macht, um nicht von der Gesamtkomplexität des Phänomens Gesellschaft überfordert zu werden. Von daher ist die hier vorgeschlagene Rekonstruktion - genauer: der hier vorgeschlagene Rekonstruktionsrahmen für eine einzelne Theorie - zum einen eine analytische Generalisierung der bereits etablierten Mikro-Makro Distinktion; zum anderen trägt die Charakterisierung der Ebenen als Emergenzebenen - die wie Mikro- und Makroebenen nicht logisch aufeinander reduzibel sind -, der immer wieder konstatierten Tatsache Rechnung, daß soziologische Theorien zwar nicht deduktionslogisch operieren können, wie jedoch jede Theorie das Besondere durch Rekurs auf ein Allgemeines erklären können müssen. Die hier postulierte Grundstruktur macht erklärlich, warum dies - wie unvollkommen auch immer - zumindest ansatzweise auch geleistet wird.

Wenn oben soziologische Theorien als vertikal geschichtete Systeme bezeichnet wurden, dann soll damit natürlich nicht gesagt werden, daß jede soziologische Theorie eine mehr oder weniger verkappte Systemtheorie wäre. Gemeint ist damit,

${ }^{13}$ Für die (Luhmannsche) Systemtheorie drückt Tyrell (1983: 76) das Konzept der empirischen Kontingenz noch wesentlich schärfer aus: ,,... jede Systemebene ist gewissermaßen unmittelbar zu Gott, für jede gelten spezifische, je unterschiedliche Möglichkeitsspielräume, Zeithorizonte und konstitutionelle Restriktionen. ... Interaktions- und Organisationssysteme (erschöpfen sich nicht) darin, Elemente oder Teile des Gesellschaftssystems zu sein, wie wohl ihre Limitierung von dort her niemals unerheblich ist." (zitiert nach Kiss 1990: 34) 
daß es sinnvoll ist, zur formalen Analyse soziologischer Theorien auf einige sehr einfache Mittel der formalen Systemanalyse zurückzugreifen, ohne dabei etwas über die Nähe oder Ferne der jeweiligen Theorien zu Systemtheorien zu unterstellen. Vielleicht ist dies ein Aspekt eines Gedankens, den Hondrich (1978: 324f) im Kontext des systematischen Theorievergleichs so ausdrückt, daß er das Paradigma der Systemtheorie für den aussichtsreichsten Kandidaten einer übergreifenden Theorie der Soziologie hält. Doch das sind gegenwärtig bestenfalls Spekulationen.

Soziologische Systemtheorien unterscheiden sich von anderen Theorien insbesondere darin, daß sie die Emergenzebenenstruktur der Theorie explizit angeben. Dies drückt sich bei Luhmann beispielsweise in der Unterscheidung zwischen Interaktion, Organisation und Gesellschaft aus; bei Parsons wird dies in den Differenzierungen des AGILSchemas deutlich, insofern diese immer neue Ebenen der Systembildungen bewirken. Bei anderen Theorien, die nicht ausdrücklich den systemtheoretischen Traditionen verpflichtet sind, ist das Prinzip der Emergenzebenen nicht so explizit, wenn auch ohne wesentliche Rekonstruktionsprobleme erkennbar: Auf den nicht nur in dieser Hinsicht logisch besonders transparenten Fall der Theorie des Historischen Materialismus habe ich bereits verwiesen; man kann a priori annehmen, $\mathrm{da} B$ Theorien, die sich dieser Tradition zuordnen, entsprechende Strukturen aufweisen. In der Habermasschen Theorie des Kommunikativen Handelns läßt sich dies leicht an der Übernahme wesentlicher Pinzipien des Marxschen Theorieansatzes demonstrieren (vgl. auch Habermas 1976). Im Fall von Mikrotheorien ist es gewöhnlich so, daß diese selbst zumindest die Distinktion zwischen einer Mikroebene als ihre Ebene $E_{1}$ und mindestens einer weiteren Makroebene thematisieren (vgl. z. B. Esser 1990).

Ich habe bisher Mikro- und Makrotheorien praktisch symmetrisch thematisiert (s. allerdings Anm. 12). In der Perspektive des hier entwickelten Rekonstruktionsmodells zeigen sich allerdings sofort einige wesentliche Unterschiede, die vielleicht plausibel machen, warum die Differenzen zwischen Mikro- und Makrotheoretikern als häufig unüberbrückbare Gegensätze erscheinen. Diese Unterschiede lassen sich folgendermaßen charakterisieren:

Bei Makrotheorien ist die Basisebene $\mathrm{E}_{1}$ stets festgelegt durch die Hypostasierung sehr allgemeiner Basisbegriffe und ebenfalls sehr allgemeiner Rela- tionen zwischen den Basisbegriffen. Die dadurch thematisierten Sachverhalte sind - jedenfalls bezogen auf konkret-sinnlich erfahrbare soziale Realität - verhältnismäßig abstrakt. Mit aller gebotenen Vorsicht läßt sich hier eine formale Parallele herstellen zu den von Chomsky (z. B. 1969) eingeführten Unterscheidungen von Tiefenstruktur und Oberflächenstruktur (vgl. zu dieser Parallele auch Habermas 1976): Die Basisebene legt für die gesamte Theorie eine Tiefenstruktur fest, die sich strukturell durch die einzelnen Ebenen durchhält; eine Ebene $E_{k}$ ist dann Oberflächenstruktur zur Basisebene $E_{1}$ und allen Ebenen $E_{i}$ mit $i<k ; E_{k}$ ist (relative) Tiefenstruktur für alle Ebenen $E_{j}$ mit j $>$ k. Der Chomskysche Begriff der Performanz entspricht dann dem, was oben als empirische Kontingenz der Ebenen $E_{i}$ mit $i>1$ bezüglich $E_{1}$ und allen Ebenen $E_{j}$ mit $1<j<i$ bezeichnet wurde.

Wieweit diese formale Parallele trägt, soll hier gar nicht weiter diskutiert werden, auch wenn ich glaube, daß mit diesen Begriffen fundamentale Charakteristika der geistes- und sozialwissenschaftlichen Theoriebildung angesprochen werden. Deutlich geworden dürfte daran sein, daß Makrotheorien „,von oben nach unten“ - wenn man $E_{1}$ als „oben" annimmt - empirisch immer reicher werden, also bezüglich ihres empirischen Gehalts räumlich als Pyramide vorzustellen sind. Gleichzeitig freilich reduziert sich die Totalität, mit der die Basisbegriffe die jeweiligen Ebenen konstituieren: Sie gelten auf „unteren“ Ebenen im wachsendem Maße nur noch partiell, da auf jeder neuen Ebene - wieder von oben nach unten fortschreitend betrachtet - neue Konstitutions- und Erklärungsprinzipien dazukommen.

Bei Makrotheorien ist $E_{1}$ als „Tiefenstruktur“ nie auch die empirische Basis, also die im Popperschen Sinne Bestätigungsebene. Deswegen existiert bei Makrotheorien so etwas wie ein eingebauter Zwang zur strukturellen Vollständigkeit, der zumindest tendenziell eingelöst werden muß: Die Basisebene $E_{1}$ ist empirisch gesehen immer unvollständig und verlangt per definitionem nach Ergänzungen bis hin zu einer Ebene $E_{n}$, die als Bestätigungsebene dienen kann - also irgendeine Mikroebene. Je abstrakter und allgemeiner die Basisebene angelegt ist, desto mehr Ebenen müssen in die Theorie eingebaut werden, um diese Ebene $E_{n} z u$ erreichen. Eine Makrotheorie, die auch tendenziell nicht strukturell vollständig ist, ist von daher entweder unempirisch oder hat ihre Basisebene nicht "universal" (Luhmann) angelegt, ist also ver- 
allgemeinerungsbedürftig. Ausgebaute Makrotheorien können aus diesem Grund gewöhnlich als strukturell vollständig bezeichnet werden - ungeachtet struktureller Lücken, empirischer Vagheiten oder auch partieller Unstimmigkeiten, die wohl bei allen existierenden Makrotheorien zu verzeichnen sind.

Bei Mikrotheorien verhält es sich in diesen Hinsichten, wie zu erwarten, fast umgekehrt. Hier ist von der Logik der Theoriebildung her die Basisebene $\mathrm{E}_{1}$ gleichzeitig auch die empirische Basis, so daß Mikrotheorien praktisch gar nicht unempirisch sein können. Dem entspricht ein in der interpretativen Sozialforschung häufig geäußertes Bedürfnis, die Komplexität der sozialen Realität möglichst vollständig auf der Basisebene zu erfassen Makrotheorien reduzieren bekanntlich durch die konstituierenden Basisbegriffe sehr selektiv (vgl. z.B. Soeffner 1989: 65 u. 185f). Diesem Vorzug der etablierten Mikrotheorien korrespondiert allerdings ein theoretischer Nachteil, da die Wahl der Basisbegriffe - Handlung, Interaktion etc. kaum Vorgaben darüber ermöglicht, wie die nächsten, abstrakteren Ebenen durch die Basisebene bestimmt werden sollen. Streng genommen ist jede Fortsetzung möglich: Eine Ebene $\mathrm{E}_{\mathrm{k}}$ mit $\mathrm{k}>1$ ist sowohl empirisch ärmer als jede Ebene $E_{i}$ mit $i<k$ als auch nur durch ad hoc Optionen mit $E_{i}(1<\mathrm{i}$ $<\mathrm{k}$ ) zu verbinden. Dies dürfte auch der Grund dafür sein, daß es gegenwärtig so gut wie keine strukturell vollständigen Mikrotheorien gibt; die Versuche von Knorr-Cetina (1981 a) beispielsweise, Mikroanalysen durch Einbezug theoretischer Makrotheorien sozusagen „nach oben“ zu ergänzen, zeigt in der den Eklektizismus streifenden Heterogenität der Begriffswahl deutlich, wie hier mit ad hoc Optionen gearbeitet werden muß.

Prinzipiell hätte eine strukturell vollständige Mikrotheorie ebenfalls die Form einer Pyramide, wobei $E_{1}$ jetzt die Pyramidenbasis bildet und die immer unempirischeren Ebenen $\mathrm{E}_{\mathrm{i}}$ sich inhaltlich nach oben „verjüngen“, also abstrakter und somit selektiver werden. ${ }^{14} \mathrm{Ob}$ es freilich überhaupt sinnvoll ist, von Mikrotheorien strukturelle Vollständigkeit zu verlangen, sei dahin gestellt; möglicher-

${ }^{14}$ Mit dem Prinzip der „abnehmenden Abstraktion“ postuliert der Mikro- bzw. Individualtheoretiker Esser einen sehr ähnlichen Gedanken. So fordert er ausdrücklich eine „Abkehr von einer „reinen" MakroAnalyse, als jede ... Vertiefung die Einführung einer Ebene „unterhalb“ des betrachteten strukturellen oder prozessualen Phänomens erfordert." (1990: 45) weise handelt es sich bei Mikrotheorien um eine soziologische Neuauflage der aus den Grundlagenkontroversen der Mathematik bekannten (intuitionistischen und konstruktivistischen) Versuche, auf der Basis individueller Bewußtseinsakte bzw. Handlungen makrotheoretisch konstituierte Bereiche zu rekonstruieren. Die Wissenschaftsgeschichte lehrt, daß derartige Versuche sowohl ungemein mühsam als auch wenig durchsetzungsfähig sind (Klüver 1971).

Hier soll jedoch von derartigen Problemen abstrahiert werden. Unterstellt man einmal die prinzipielle Möglichkeit, sowohl strukturell vollständige Mikro- als auch Makrotheorien zu entwickeln, dann stellen sich vor allem zwei Probleme für die Theoriekonstruktion. Zum einen ist dies ein sozusagen doppeltes Relationenproblem: Die Theorie muß sowohl Relationen zwischen den einzelnen Emergenzebenen postulieren, um überhaupt einen Theoriezusammenhang zu erhalten, als auch Relationen auf den einzelnen Ebenen angeben, da sonst kaum von Emergenzebenen die Rede sein kann. Wenn man die ersteren als vertikale und die letzteren als horizontale Relationen bezeichnet, dann drückt sich in der Wahl der vertikalen Relationen aus, wie die Theorie durch die Basisebene die Gegenstandsbereiche der anderen Ebenen, also der gesamten Theorie, kategorial konstituiert und damit „erklärt"; die Wahl der horizontalen Relationen drückt sowohl aus, wie sich die Basisrelationen auf den anderen Ebenen reproduzieren das ist der Teil der Relationen von $\mathrm{E}_{\mathrm{k}}$, der mit den Relationen von $E_{1}$ identisch ist -, als auch, was als empirische Kontingenz auf $E_{k}$ strukturell hinzukommt. Man muß also bei den horizontalen Relationen - und evtl. auch bei den vertikalen - davon ausgehen, daß die Anzahl der Relationen von Ebene zu Ebene differiert: Die Menge der Relationen auf $E_{k}(k>1)$ ist größer als die auf $E_{k-1}$, falls es sich um eine Makrotheorie handelt, sonst umgekehrt. Aus logischen und ästhetischen Gründen gibt es sicher häufig Bemühungen, die Anzahl der vertikalen und horizontalen Relationen möglichst gering zu halten.

Auch hierfür liefert die Theorie des Historischen Materialismus ein illustratives Beispiel. Wenn man die protosoziologische Basisebene der Arbeit nicht berücksichtigt, erhält man auf der ersten ,soziologischen" Ebene die dialektische Beziehung zwischen Produktivkräften und Produktionsverhältnissen. Diese horizontale Relation setzt sich als Verhältnis zwischen Basis und Überbau fort, wobei sie auf dieser Emergenzebene eine Mehrfach- 
funktion hat: Sie beschreibt auch das Verhältnis in der Zeit zwischen verschiedenen historischen Gesellschaftsformationen, die bekanntlich durch dialektische Widersprüchlichkeit auseinander hervorgehen. Entsprechend existiert eine dialektische Beziehung zwischen den einzelnen Klassen. Hier setzt sich also eine Relationenstruktur konstant durch die Ebenen fort. Die vertikalen Relationen lassen sich in diesem Theoriefall als Konstitutionsprinzipien charakterisieren: Arbeit $\left(\mathrm{E}_{1}\right)$ konstituiert sich gesellschaftlich als Produktivkraft und Produktionsverhältnis; diese konstituieren sich als Basis, der dialektisch dann der Überbau ergänzend zugeordnet wird, entsprechend konstituieren sich Klassen als politische Grundstruktur (vgl. etwas anders orientiert auch die Rekonstruktion bei Habermas 1976). ${ }^{15}$ Allgemein zeigen Alexander/Giesen aaO. und Giesen aaO. in ihren Übersichten, welche vertikalen Relationen möglich sind und auch in den einzelnen Theoriemodellen realisiert werden.

Das zweite Problem betrifft ebenfalls die Beziehungen zwischen den einzelnen Emergenzebenen und zeigt, inwiefern die traditionelle Mikro-Makro-Distinktion wesentlich als analytische Unterscheidung betrachtet werden muß. Münch/Smelser (1987) weisen m.E. korrekt darauf hin, daß es sozusagen „reine“ Makro- und Mikrotheorien in einem bestimmten Sinne gar nicht geben kann: Beide Theorieansätze sind grundsätzlich darauf angewiesen, Rückgriffe beim jeweils anderen Theorieansatz zu machen. So können Mikroansätze nicht umhin, entgegen ihrer Grundorientierung mikroübergreifende Handlungsprinzipien zu hypostasieren, die nur aus Makroansätzen heraus begründbar sind. Entsprechend sind Makroansätze darauf angewiesen, sich Gesetzmäßigkeiten auf den Mikroebenen zu vergewissern, die dann auf Makroebenen zurückwirken. Es lassen sich also weder Konstitutionen von unten noch von oben streng durchhalten.

In systemtheoretischen Begrifflichkeiten liegt hier, wenn man der Charakterisierung von Theorien als vertikal geschichtete Systeme folgt, offensichtlich ein bekanntes Phänomen vor: Anscheinend muß zwischen den einzelnen Ebenen so etwas wie eine Rückkopplungsschleife angenommen werden, um die komplexen Interdependenzen zwischen den verschiedenen Ebenen zu erfassen. Es reicht also

${ }^{15} \mathrm{Vgl}$. in diesem Zusammenhang auch die am Sneedschen Formalismus orientierte Rekonstruktion von Teilen der Marxschen Theorie bei Diederich 1981. nicht, nur jeweils einseitig gerichtete (konstituierende) vertikale Relationen „von oben nach unten" oder umgekehrt anzunehmen, sondern soziologische Theorien sind - implizit - immer schon so komplex wie die von ihnen analysierte und konstituierte Realität: Die aus der allgemeinen Systemtheorie und speziell der Theorie dynamischer Systeme hinlänglich bekannte Tatsache, daß komplexe Systemzusammenhänge nicht in linearen unidirektionalen Relationen beschrieben werden können, findet in der implizit vorgegebenen Architektonik soziologischer Theoriebildung eine indirekte Bestätigung.

Noch einmal sei in diesem Zusammenhang Marx zur Illustration herangezogen: „... die verschiedenen Momente des Überbaus - politische Formen des Klassenkampfes und seine Resultate - ... und nun gar die Reflexe aller dieser wirklichen Kämpfe im Gehirn der Beteiligten ... üben auch ihre Einwirkung auf den Verlauf der geschichtlichen Kämpfe aus und bestimmen in vielen Fällen deren Form" (MEW 37: 463; vgl. auch Bader u. a. 1987: 58). Für die Erklärung sozialen Wandels, so läßt sich diese Stelle offenbar interpretieren, kommt die historisch-materialistische Makrotheorie nicht ohne Rekurs auf eine Wirkungsbeziehung von Mikroebenen auf Makroebenen aus; anscheinend ist die Erklärung sozialen Wandels immer der kritische Punkt, bei dessen Bearbeitung sowohl Makro- wie Mikrotheorien ihre Orientierungen ändern müssen (vgl. Münch/Smelser 1987). In der hier verwendeten Rekonstruktionssprache verweist diese Stelle auf eine Rückkoppelungsschleife innerhalb der Theorie: Die Makroebenen Basis/ Überbau und Klassenstruktur konstituieren die Mikroebene des individuellen Bewußtseins, indem sie als Ausdruck des „Seins das Bewußtsein bestimmen" (MEW 13: 8f), und es gleichzeitig produzieren. Die Mikroebene wirkt demgegenüber auf die Makroebenen ein, indem das Bewußtsein die Klassenkämpfe (mit)beeinflußt, deren Ergebnis dann bekanntlich eine neue Gesellschaftsformation ist. Rückkopplungsschleifen zwischen den einzelnen Emergenzebenen sind vermutlich wesentlicher Bestandteil jeder elaborierten Theoriestruktur; damit verlieren jedoch die traditionellen Mikro-Makro-Distinktionen ihre Schärfe.

Zur Illustration dieser Rekonstruktionsüberlegungen soll zum Abschluß dieses Abschnittes noch einmal das Beispiel des Historischen Materialismus herangezogen werden, die als klassische Makrotheorie bei aller bekannten empirischen und begrifflichen Unzulänglichkeit nach wie vor als 
Exempel für eine tendenziell strukturell vollständige Theorie dienen kann. Das folgende Schema faßt die bisherigen Überlegungen graphisch zusammen und braucht wohl nicht weiter erläutert zu werden.

1. 〈(Arbeit) (Mensch) "=" Gattung

2. 〈(Produktivkräfte R Produktionsverhältnisse) $"="$ Produktionsweise $\rangle$

3. $\left\langle\left(\text { Basis }_{i} R \text { Überbau }\right)_{2}\right)="$ Gesellschaftsformation $\rangle$

3.1. (Kapitalismus $R_{1}$ Feudalismus etc.)

3.2. (Recht $R_{i}$ Kultur $R_{i}$ Religion etc.)

4. ((Herrschende Klasse $R$ produzierende Klasse) "=" Sozialstruktur $\rangle$

4.1. (Sklavenhalter $R_{i}$ Feudalisten $R_{i}$ Kapitalisten (Bürger))

4.2. (Sklaven $\mathbf{R}_{i}$ (leibeigene) Bauern $R_{i}$, Proletarier)

4.3. (Antike Hochkultur $R_{i}$ mittelalterliche Ständegesellschaft $R_{i}$ bürgerliche (moderne) Gesellschaft)

5. 〈(Interklassenbeziehung R Intraklassenbeziehungen) $"=$ " Sozialbeziehungen $\rangle$

5.1. Warenbeziehung etc.

5.2. Proletarische Solidarität etc.

(bei Marx vermutlich systematische Lücke, daher Thema der kritischen Theorie als Bindeglied zwischen 4. und 6.)

6. 〈(Klassenbewußtsein (Ideologie) $\mathrm{R}$ richtiges Bewußtsein " $=$ "Bewußtsein $\rangle^{16}$

\section{Metatheoretische Konsequenzen und wissensbasierte Systeme}

Die Aufgabe, einen metatheoretischen Vergleichsrahmen für unterschiedliche Theorien zu konstruieren, läßt sich aufgrund der hier vorgetragenen Generalisierung der Mikro-Makro-Distinktionen jetzt folgendermaßen formulieren und teilweise auch beantworten: Grundlegend ist die Annahme, $\mathrm{da} ß$ sich soziologische Theorien als vertikal geschichtete "Systeme" betrachten lassen, deren

${ }^{16}$ Mir ist bewußt, daß Marx diese Gesamttheorie nie ausgearbeitet hat und daß lediglich die programmatischen Frühschriften die gesamte Theorie thematisieren. Es handelt sich hier also um so etwas wie „einen ideellen Gesamtmarx“. Die hier rekonstruierte Theoriearchitektur läßt sich freilich auch in den Analysen der bürgerlichen Gesellschaft auffinden (vgl. auch Lange 1980), wie erste Rekonstruktionen des „Kapital $^{\text {“ }}$ auf der Basis dieses Schemas bereits gezeigt haben.
Schichtungen aus den Emergenzebenen bestehen und durch vertikale wie horizontale Relationen gekennzeichnet werden können; die vertikalen Relationen können (und werden häufig) auch in Form von Rückkopplungsschleifen auftreten.

Ein Vergleich zweier Theorien besteht in dieser sehr formalen Weise darin, die Theorien jeweils diesem Schema zuzuordnen: Identifizierung der jeweiligen Basisebene sowie der übrigen in der einzelnen Theorie enthaltenen Emergenzebenen, Rekonstruktion der wesentlichen vertikalen und horizontalen Relationen einschließlich der Rückkopplungsschleifen, kurz: Vergleich der Theorien auf deren jeweilig strukturelle Vollständigkeit und damit ihre formale Leistungsfähigkeit. Ein derartiger formaler Vergleich der Theoriearchitekturen ist natürlich in keiner Weise hinreichend dafür, $\mathrm{zu}$ entscheiden, ob einer in dieser Hinsicht formal überlegenen Theorie eindeutig der Vorzug gegenüber ihren Konkurrentinnen gegeben werden soll. So geht es bei Theorienkonkurrenz noch nicht einmal in der Mathematik zu. Zumindest gegenwärtig ist die in Abschnitt 2 angesprochene Weltbildfunktion soziologischer Theorien wohl ebenso entscheidend über ihre Akzeptierbarkeit wie ihre formale Leistungsfähigkeit. Ein solcher Formalvergleich kann jedoch darüber Aufschluß geben, inwieweit es sich bei den einzelnen Theorien überhaupt um strukturierte Gebilde handelt, die diesen Namen verdienen, nämlich um logisch geordnete Komplexe, die formalen Analysen zugänglich sind und die ihre Erklärungsfähigkeit, sofern sie eine haben, aus überprüfbaren Beziehungen einzelner Phänomene auf allgemeine Zusammenhänge gewinnen. Eine derartige Anforderung an soziologische Theorien zu stellen, ist gewiß nicht zuviel verlangt; im theoretischen Einzelfall den entsprechenden positiven oder auch negativen Nachweis zu führen, ist allerdings alles andere als trivial.

Zur Unterstützung der formalen Rekonstruktion einzelner Theorien haben wir deshalb vorgesehen, spezielle wissensbasierte Systeme, also Computerprogramme, $z u$ entwickeln, die eine detaillierte Rekonstruktion und einen entsprechend detaillierten Vergleich überhaupt erst ermöglichen sollen. Wie diese wissensbasierten Systeme grundsätzlich entwickelt werden und operieren sollen, wird in späteren Veröffentlichungen dargestellt werden (vgl. Klüver/Krallmann 1989).

Der Begriff eines „computerunterstützten Theorienvergleichs" besagt in diesem Kontext, daß (a) die logischen Grundstrukturen einzelner Theorien rekonstruiert werden gemäß dem metatheoreti- 
schen Strukturrahmen, (b) für jede rekonstruierte Theorie ein spezielles Programmsystem, ein sog. wissensbasiertes System, entwickelt wird, das als Bestandteile seiner Wissensbasis u.a. die rekonstruierten Theoriestrukturen enthält, und daß (c) ein „Metasystem“ als wissensbasiertes System entwickelt wird, das formale Strukturvergleiche zwischen den einzelnen rekonstruierten Theorien genauer: ihrer Implementation in den theoriespezifischen wissensbasierten Systemen - durchführen kann. Das kann hier aus Raumgründen nicht weiter erläutert werden. Hier ging es erst einmal um die Darstellung des Weges, wie soziologische Theorien logisch rekonstruiert werden können, um zur Basis spezieller Programmsysteme zu werden. Ich glaube, daß entscheidenden Prinzipien soziologischer Theoriebildung damit Rechnung getragen werden und daß diese so auch erst transparent werden.

\section{Literatur}

Adorno, T. W. (Hrsg.), 1970: Der Positivismusstreit in der deutschen Soziologie. Neuwied

Alexander, J. C., 1987: Action and Its Environments. In: Alexander u. a.

Alexander, J. C./Giesen, B./Münch, R./Smelser, N. J. (Hrsg.), 1987: The Micro-Macro Link. Berkeley-Los Angeles-London

Alexander, J. C./Giesen, B., 1987: From Reduction to Linkage: The Long View of the Micro-Macro Link. In: Alexander u. a.

Bader, V. M./Berger, J./Ganßmann, H./v.d. Knesebeck, J., 1987: Einführung in die Gesellschaftstheorie. ${ }^{2}$ Frankfurt-New York

Bayer, O./Stölting, E., 1989: Stichwort Sozialwissenschaften. In: Seiffert, H./Radnitzky, G. (Hrsg.): Handlexikon zur Wissenschaftstheorie. München

Carnap, R., 1950: Empirism, Semantics and Ontology. In: Revue Internationale de Philosophie II

Chomsky, N., 1969: Aspekte der Syntax Theorie. Frankfurt

Cohen, P. S., 1980: Stichwort Soziologische Theorie. In: Speck, J. (Hrsg.): Handbuch wissenschaftstheoretischer Begriffe, Bd. 3, Göttingen

Diederich, W., 1981: Strukturalistische Rekonstruktionen. Braunschweig-Wiesbaden

Esser, H., 1990: Alfred Schütz und "rational choice“. Mskr. Köln

Fielding, N. G. (Hrsg.), 1989: Actions and Structure. Research Methods and Social Theory. London

Geulen, D./Hurrelmann, K., 1980: Zur Programmatik einer umfassenden Sozialisationstheorie. In: Hurrelmann, K./Ulich, D. (Hrsg.): Handbuch der Sozialisationsforschung. Weinheim-Basel

Giesen, B., 1987: Beyond Reductionism: Four Models Relating Micro and Macro Levels. In: Alexander u. a.
Habermas, J., 1976: Zur Rekonstruktion des Historischen Materialismus. Frankfurt

Habermas, J., 1981: Theorie des kommunikativen Handelns, II, Frankfurt

Hannemann, R. A./Collins, R., 1986: A dynamic simulation of Marx's model of capitalism. In: Wiley, $\mathbf{N}$. (Hrsg.): The Marx/Weber Debate. Newbury Park

Hannemann, R. A., 1988: Computer Assisted Theory Building. Modeling Dynamic Social Systems. Newbury Park-Beverly Hills-London-New Dehli

Hondrich, K. O., 1978: Viele Ansätze - eine soziologische Theorie. In: Hondrich/Matthes

Hondrich, R. O./Matthes, J. (Hrsg.), 1978: Theorievergleich in den Sozialwissenschaften. Darmstadt-Neuwied

Janisch, P., 1969: Die Protophysik der Zeit. Mannheim

Kiss, G., ' 1990: Grundzüge und Entwicklung der Luhmannschen Systemtheorie. Stuttgart

Klinkmann, N., 1981: Der systematische Vergleich von Theorien. Ein Versuch und die Unausweichlichkeit seines Scheiterns. In: Soziale Welt 32: 249-260

Klüver, J., 1971: Operationalismus. Geschichte und Kritik einer Philosophie der exakten Wissenschaften. Stuttgart

Klüver, J./Müller, W., 1972: Wissenschaftstheorie und Wissenschaftsgeschichte: Die Entdeckung der Benzolformel. Zeitschrift für allgemeine Wissenschaftstheorie III

Klüver, J./Krallmann, D., 1989: Entwicklung eines wissensbasierten Systems zum Vergleich formal rekonstruierter soziologischer Theorien. Mskr. Essen

Knorr, K. D./Krohn, R./Whitley, R. (Hrsg.) 1980: The Social Process of Scientific Investigation. DordrechtBoston-London

Knorr-Cetina, K./Cicourel, A. V. (Hrsg.), 1981: Advances in Social Theory and Methodology. BostonLondon

Knorr-Cetina, K., 1981: The micro-sociological challenge of macro-sociology: towards a reconstruction of social theory and methodology. In: Knorr-Cetina/Cicourel

Knorr-Cetina, K. D., 1981 (=1981 a): The Manufacture of Knowledge. Oxford

Krohn, W./Küppers, G., 1989: Die Selbstorganisation der Wissenschaft. Frankfurt

Krohn, R., 1980: Toward the Empirical Study of Scientific Practice. In: Knorr/Krohn/Whitley

Lange, E. M., 1980: Das Prinzip Arbeit. FrankfurtBerlin-Wien

Lepsius, R. (Hrsg.), 1976: Zwischenbilanz der Soziologie. Stuttgart

Lorenzen, P., 1962: Metamathematik. Mannheim

Luckmann, T., 1980: Lebenswelt und Gesellschaft. Paderborn

Luhmann, N., 1984: Soziale Systeme. Frankfurt

Minsky, M., 1975: A Framework for Representing Knowledge. In: Winston, P. H. (Hrsg.): The psychology of Computer Vision. New York

Münch, R., 1988: Theorie des Handelns. Frankfurt

Münch, R./Smelser, N. J., 1987: Relating the Micro and Macro. In: Alexander u. a. 
Opp, K. O./Wippler, R., 1990: Theoretischer Pluralismus und empirische Forschung. In: Dies. (Hrsg.): Empirischer Theorienvergleich. Opladen

Pickering, A. R., 1980: The role of Interest in HighEnergy Physics: The Choice between Charm and Colour. In: Knorr/Krohn/Whitley
Soeffner, H. G., 1989: Auslegung des Alltags. Der Alltag der Auslegung. Frankfurt

Tyrell, H., 1983: Zwischen Interaktion und Organisation I: Gruppe als Systemtyp. In: Sonderheft der KZfSS 25 